\title{
Lung stem cells - from an evolving understanding to a paradigm shift?
}

Mairéad Hayes ${ }^{1,2}$, Gerard F Curley ${ }^{1,2}$ and John G Laffey ${ }^{* 1-3}$

\begin{abstract}
The ideal cell type to regenerate an acutely injured or chronically diseased lung would be a stem cell population from the patient's own lung. Consequently, extensive research efforts have focused on identifying and characterizing endogenous lung stem cells. Advances in techniques to facilitate cell isolation, labelling and tracking in vivo to determine their fate have led to the identification of several putative stem cell niches. Recently, convincing evidence has emerged for a novel stem/progenitor cell population in the submucous glands of the cartilaginous airways. These findings support the concept that there is no classical stem cell 'hierarchy' but that different progenitor populations within spatially distinct lung regions regenerate the lung epithelium adjacent to its niche. Intriguingly, recent findings challenge this concept; it was reported that the human lung may contain a primitive stem cell capable of differentiating into multiple cells of both endodermal and mesodermal lineage and of regenerating the injured lung. This suggests that a classical stem cell hierarchy may, in fact, exist in the lung. Although caution is needed in interpreting these emerging findings, the implications for our current concepts regarding lung stem cells, the insights into lung repair and regeneration, and the potential therapeutic implications are considerable.
\end{abstract}

\section{Background}

Respiratory diseases such as chronic obstructive pulmonary disease, asthma pulmonary fibrosis, and acute lung injury/acute respiratory distress syndrome (ALI/ARDS) confer an enormous disease burden. Despite extensive research efforts, curative therapies for these diseases have

*Correspondence: john.laffey@nuigalway.ie

'Lung Biology Group, Regenerative Medicine Institute, National Centre for Biomedical Engineering Science, National University of Ireland, University Road, Galway, Ireland

Full list of author information is available at the end of the article proven elusive and current management remains limited to symptom control or supportive strategies or both. A promising therapeutic approach may be the use of stem cells to aid lung regeneration and repair. Although exogenous stem cells, particularly mesenchymal stem cells, have received much attention [1], lung stem cell populations might be the ideal cell to regenerate the lung [2]. Progress in this area has been difficult [3], but recent advances have invigorated this field.

\section{Insights from lung development}

The lung, being derived from cells of endodermal and mesodermal origin, has a complex embryological origin. Elegant studies, in which individual cells in the developing embyro are labeled to allow their descendants (that is, lineage) to be traced, have generated considerable insights. Lung development begins during the fifth gestational week with the formation of epithelial buds from the primordial endoderm which then grow into the embryonic mesenchyme. Almost all of the epithelial cell types found in the developed lung arise from these earliest lung-committed buds [4]. In a complex but poorly understood process involving much crosstalk between the dermal layers, these buds organize to form the epithelial architecture, whereas the mesoderm forms the supporting stroma and blood vessels [5]. Of importance, there is no evidence from lineage-tracing studies that the embryonic lung - or, indeed, any developing organ - contains a common progenitor for endodermal and mesodermal lineages [6,7]. This suggests that separate populations of stem/progenitor cells are necessary for the maintenance of the endodermal- and mesodermal-derived lung components.

\section{Insights from other organs}

Mammalian tissue regeneration appears to involve several mechanisms, including compensatory hyperplasia, dedifferentiation, and adult stem cells. Different organs use different strategies to renew themselves, and more diversity and flexibility underpin these renewal processes than previously imagined. Some organs, such as hair follicles, blood, and gut, which constantly renew themselves throughout life, contain adult stem cells that are 
morphologically unspecialized, have a relatively low rate of division, and are topologically restricted to localized regions known as 'niches' that tightly regulate their behavior [8]. In the intestine, for example, only a few stem cells are present near the base of the crypts, and these stem cells appear to be responsible for replenishing the entire epithelium. In contrast, the adult lung normally regenerates slowly, but following lung injury, rapid repair is essential for survival.

\section{Current concepts of lung tissue regeneration}

Current paradigms propose the existence of a 'nonclassical' stem cell hierarchy in the lung, in which relatively quiescent progenitor cells differentiate into a restricted number of cells to regenerate specific lung epithelial cells and structures [9]. Advances in laboratory techniques that facilitate in vivo cell isolation, labeling, and tracking have identified several candidate stem cell populations (Figure 1). These putative microenvironmental niches appear to contain cells that can self-renew and generate differentiated progeny that regenerate the lung epithelium adjacent to its respective niche $[5,10]$. In the trachea and main bronchi, it appears that undifferentiated basal cells can function as classical stem cells, both self-renewing and giving rise to ciliated and secretory cells. Duct cells from submucosal glands (SMGs) located in the cartilaginous airways are also a potential niche [10]. In the more distal lung, Clara cells [2] and subpopulations of Clara cells (termed 'variant' Clara cells) found in the bronchioles and bronchiolar-alveolar junctions, respectively, appear to self-renew and give rise to different cell types after injury [10]. In the alveoli, damaged type I cells can be generated from dividing type II cells, although whether all type II cells have this capacity is not yet known [5].

The concept that there are different progenitor populations within spatially distinct regions in the adult lung, rather than a 'master' lung stem/progenitor cell capable of repairing any lost epithelial cell, is further supported by recent fate-mapping studies $[11,12]$. This regeneration model may reflect the special requirements for lung regeneration and repair, in which the ability of multiple cell types to proliferate and rapidly repair the lung may be advantageous.

\section{The submucosal gland duct stem/progenitor cell}

Previous studies have suggested that SMGs might constitute a stem cell niche, and progenitor cells have been identified in the SMGs of other organs, including the breast and submandibular gland $[13,14]$. Now, Hegab and colleagues [15] provide convincing evidence that SMG duct cells do constitute a stem/progenitor cell population. These investigators developed a novel approach to label and isolate these cells. These SMG duct cells survived in vivo during hypoxic-ischemic injury and were capable of self-renewal and of differentiation into SMG duct and tubule cells and surface epithelium cells [15]. Though sharing similarities with basal cells, these SMG duct cells appear to be a distinct stem/progenitor cell population.

As we consider these findings, it is important to remember that the anatomy and physiology of the murine proximal airway differ significantly from those of humans [10]. Specifically, human SMG cells are more copious and are found throughout the cartilaginous airways rather than in just the upper third of the trachea and larynx. Also, interspecies variation in SMG location between different strains of inbred mice has been reported [16]. Consequently, these findings need to be replicated in the human lung. It is also unclear whether these are true stem or progenitor cells, a common issue in studies such as this. Features that suggest that these are progenitor rather than true stem cells include their limited differentiation potential and the fact that, being capable of mucin secretion, they are already specialized.

Notwithstanding these issues, the identification of this novel stem/progenitor cell population is important for a number of reasons. First, given the position of these cells throughout the cartilaginous airways in humans, these cells may play a major role in repair following diffuse injury to the lung, such as that seen in ALI/ARDS. Second, dysfunction of these cells may contribute to the pathogenesis of a number of hypersecretory lung diseases, including chronic obstructive airway disease, asthma, and cystic fibrosis. Third, these cells express the K14 surface marker, which is seen in pre-malignant lesions and which correlates with poor prognosis in nonsmall cell lung cancer, suggesting that these cells might be the stem cell of origin of these cancers.

\section{The 'true' lung stem cell - a paradigm shift?}

Intriguingly, Kajstura and colleagues [17] now provide evidence that the lung may contain 'true' stem cells. In a series of elegant experiments, the authors demonstrate that these $\mathrm{c}-\mathrm{kit}^{+}$cells are undifferentiated, self-renewing, clonogenic, and multipotent in vitro. These undifferentiated cells do not express lung-specific proteins or hematopoietic markers. The multipotency and apparent plasticity of these cells may arise from concurrent expression of the transcription factors Nanog, Oct3/4, Sox2, and Klf-4. The finding that, when injected into a mouse model of focal lung injury, these human c-kit ${ }^{+}$ cells appeared to regenerate many different lung components - including bronchioles, alveoli, smooth muscle, and even pulmonary vessels - is as surprising as it is exciting [17].

If confirmed, these findings constitute a landmark development. The implications for our current concepts regarding lung stem cells, the insights into lung repair 


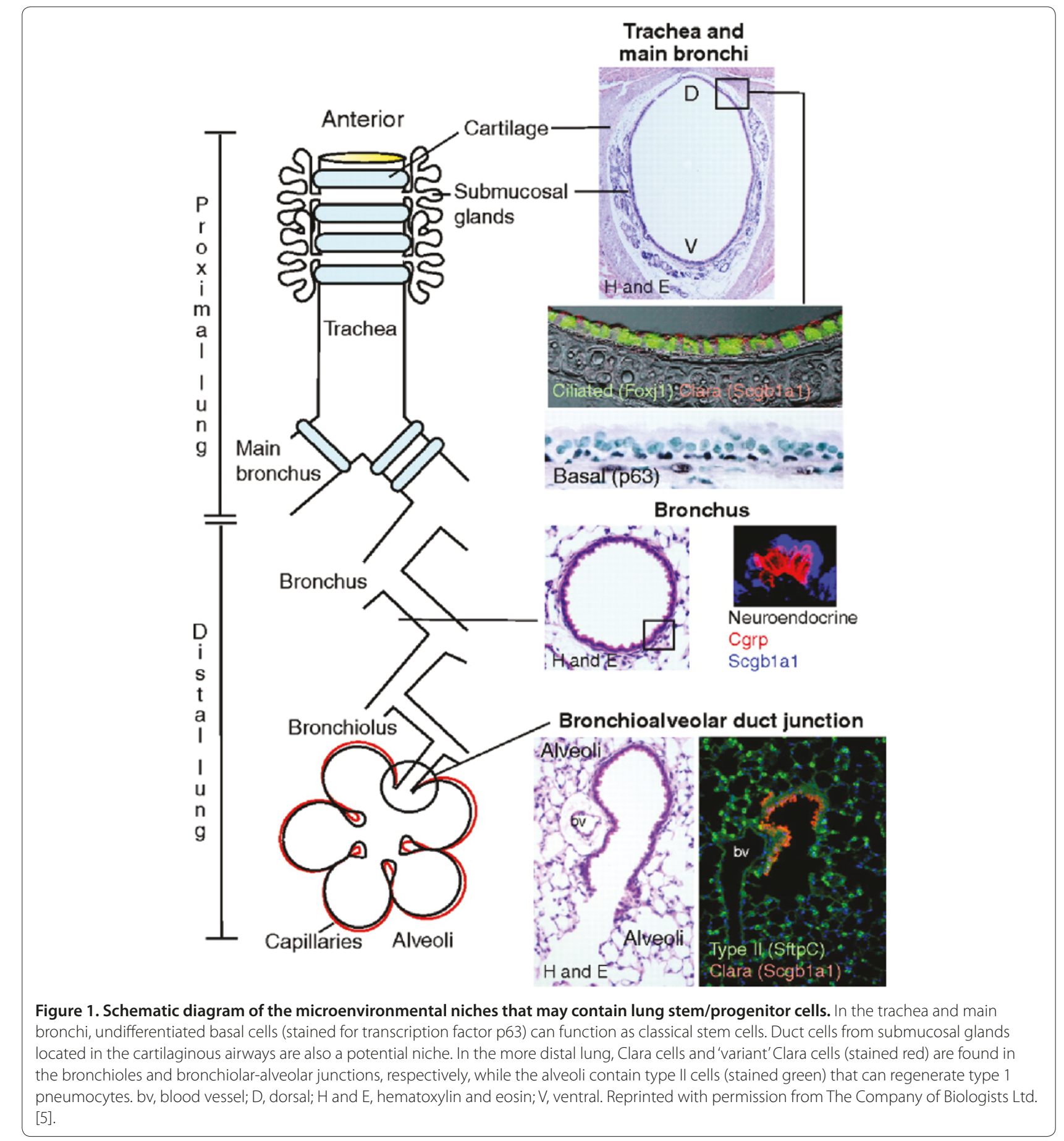

and regeneration, and the potential therapeutic implications are considerable. These $\mathrm{c}-\mathrm{kit}^{+}$lung stem cells could be used to stimulate lung tissue regeneration in a patient with lung diseases that result in loss of alveoli (such as chronic obstructive pulmonary disease) or that are characterized by widespread loss of lung epithelium (such as ALI/ARDS). These findings could bolster attempts to bioengineer lung tissue in vitro [18]. However, enthusiasm for the findings of the study must not preclude a careful appraisal of its strengths and limitations [7].

\section{Lung stem cells - where are we now?}

The findings of Kajstura and colleagues [17] pose a serious challenge to our existing concepts by providing evidence for a classical stem cell hierarchy in the adult 
lung. At the top of this hierarchy are c-Kit ${ }^{+}$cells, which constitute the ultimate reservoir for self-renewal of epithelial (and mesenchymal) progenitor cells, such as basal cells, Clara cells, and SMG duct cells. The novelty of these findings is underlined by the fact that multipotent stem cells that can give rise to both endodermal and mesodermal lineages have not previously been described in the lung or, indeed, in any organ [9]. Furthermore, there is no known interconversion of endodermal and mesodermal cells during embryonic lung development [18].

Not surprisingly perhaps, this study has already generated significant comment in the literature $[7,18]$. Issues highlighted in a discussion in Nature Medicine [7] include the fact that these studies, though extensive, lacked appropriate controls for certain key experiments; another issue is the need to more clearly define the niche of these cells within the lung and to determine the factors that regulate their quiescence and activation. These studies focused solely on human c-kit ${ }^{+}$stem cells. Subsequent studies, which will be required to validate these findings, could use more tractable systems, such as genetically modified mouse models. Detailed in vivo lineage-tracking studies of the fates of c-kit lung stem cells, during normal lung maintenance and in response to lung injury, would provide significant additional insights.

\section{Conclusions}

This is an exciting time for lung stem cell research. Newer and more robust laboratory techniques that facilitate cell isolation, labeling, and tracking to determine stem cell fate in vivo have considerably advanced our knowledge of these populations of the lung. Although the need for caution in considering the clinical implications of these findings is clear, these studies provide exciting new insights. The studies challenge our current concepts and should energize the field of lung stem cell research.

\section{Abbreviations \\ ALI/ARDS, acute lung injury/acute respiratory distress syndrome; SMG, submucosal gland.}

\section{Competing interests}

The authors declare that they have no competing interests.

\section{Acknowledgments}

This work was supported by funding from the Health Research Board, Dublin, Ireland (grant RP/2008/193), and the European Research Council, Brussels, Belgium, under the Framework 7 Programme (grant ERC-2007-StG 207777).

\section{Author details}

'Lung Biology Group, Regenerative Medicine Institute, National Centre for Biomedical Engineering Science, National University of Ireland, University Road, Galway, Ireland; '2Department of Anaesthesia, Galway University Hospitals, Newcastle Road, Galway, Ireland; ${ }^{3}$ School of Medicine, Clinical Sciences Institute, National University of Ireland Galway, Costello Road, Galway, Ireland.

Published: 20 October 2011

\section{References}

1. Moodley Y, Manuelpillai U, Weiss DJ: Cellular therapies for lung disease: a distant horizon. Respirology 2011, 16:223-237.

2. Snyder JC, Teisanu RM, Stripp BR: Endogenous lung stem cells and contribution to disease. J Pathol 2009, 217:254-264.

3. Weiss DJ, Kolls JK, Ortiz La, Panoskaltsis-Mortari A, Prockop DJ: Stem cells and cell therapies in lung biology and lung diseases. Proc Am Thorac Soc 2008, 5:637-667.

4. Copland I, Post M: Lung development and fetal lung growth. Paediatr Respir Rev 2004, 5 Suppl A:S259-264.

5. Rawlins EL, Hogan BL: Epithelial stem cells of the lung: privileged few or opportunities for many? Development 2006, 133:2455-2465.

6. Morrisey EE, Hogan BL: Preparing for the first breath: genetic and cellular mechanisms in lung development. Dev Cell 2010, 18:8-23.

7. Lung stem cells: looking beyond the hype. Nat Med 2011, 17:788-789.

8. Fuchs E, Tumbar T, Guasch G: Socializing with the neighbors: stem cells and their niche. Cell 2004, 116:769-778.

9. Stripp BR: Hierarchical organization of lung progenitor cells: is there an adult lung tissue stem cell? Proc Am Thorac Soc 2008, 5:695-698.

10. Liu X, Engelhardt JF: The glandular stem/progenitor cell niche in airway development and repair. Proc Am Thorac Soc 2008, 5:682-688.

11. Rawlins EL, Clark CP, Xue Y, Hogan BL: The Id2+ distal tip lung epithelium contains individual multipotent embryonic progenitor cells. Development 2009, 136:3741-3745.

12. Rawlins EL, Okubo T, Xue Y, Brass DM, Auten RL, Hasegawa H, Wang F, Hogan BL: The role of Scgb1a1+Clara cells in the long-term maintenance and repair of lung airway, but not alveolar, epithelium. Cell Stem Cell 2009, 4:525-534.

13. Visvader JE: Keeping abreast of the mammary epithelial hierarchy and breast tumorigenesis. Genes Dev 2009, 23:2563-2577.

14. Kimoto M, Yura Y, Kishino M, Toyosawa S, Ogawa Y: Label-retaining cells in the rat submandibular gland. J Histochem Cytochem 2008, 56:15-24.

15. Hegab AE, Ha VL, Gillbert JL, Zhang KX, Malkoski SP, Chon AT, Darmawan DO, Bisht B, Ooi AT, Pellegrini M, Nickerson DW, Gomperts BN: Novel stem/ progenitor cell population from murine tracheal submucosal gland ducts with multipotent regenerative potential. Stem Cells 2011, 29:1283-1293.

16. Innes BA, Dorin JR: Submucosal gland distribution in the mouse has a genetic determination localized on chromosome 9. Mamm Genome 2001, 12:124-128

17. Kajstura J, Rota M, Hall SR, Hosoda T, D'Amario D, Sanada F, Zheng H, Ogórek B, Rondon-Clavo C, Ferreira-Martins J, Matsuda A, Arranto C, Goichberg P, Giordano G, Haley KJ, Bardelli S, Rayatzadeh H, Liu X, Quaini F, Liao R, Leri A, Perrella MA, Loscalzo J, Anversa P: Evidence for human lung stem cells. N Engl J Med 2011, 364:1795-1806.

18. Chapman HA: Toward lung regeneration. N Eng/ J Med 2011, 364:1867-1868.

doi:10.1186/scrt82

Cite this article as: Hayes $M$, et al.: Lung stem cells - from an evolving understanding to a paradigm shift? Stem Cell Research \& Therapy 2011, 2:41. 